\title{
Synergistic Anticancer Effects of Vorinostat and Epigallocatechin-3-Gallate against HuCC-T1 Human Cholangiocarcinoma Cells
}

\author{
Tae Won Kwak, Do Hyung Kim, Chung-Wook Chung, Hye Myeong Lee, \\ Cy Hyun Kim, Young-IL Jeong, and Dae Hwan Kang
}

\begin{abstract}
National Research and Development Center for Hepatobiliary Disease, Pusan National University Yangsan Hospital, Gyeongnam 626-770, Republic of Korea
\end{abstract}

Correspondence should be addressed to Dae Hwan Kang; sulsulpul@naver.com

Received 8 January 2013; Accepted 28 May 2013

Academic Editor: Andreas Sandner-Kiesling

Copyright (C) 2013 Tae Won Kwak et al. This is an open access article distributed under the Creative Commons Attribution License, which permits unrestricted use, distribution, and reproduction in any medium, provided the original work is properly cited.

\begin{abstract}
The aim of this study was to investigate the effect of the combination of vorinostat and epigallocatechin-3-gallate against HuCC-T1 human cholangiocarcinoma cells. A novel chemotherapy strategy is required as cholangiocarcinomas rarely respond to conventional chemotherapeutic agents. Both vorinostat and EGCG induce apoptosis and suppress invasion, migration, and angiogenesis of tumor cells. The combination of vorinostat and EGCG showed synergistic growth inhibitory effects and induced apoptosis in tumor cells. The Bax/Bcl-2 expression ratio and caspase- 3 and -7 activity increased, but poly (ADP-ribose) polymerase expression decreased when compared to treatment with each agent alone. Furthermore, invasion, matrix metalloproteinase (MMP) expression, and migration of tumor cells decreased following treatment with the vorinostat and EGCG combination compared to those of vorinostat or EGCG alone. Tube length and junction number of human umbilical vein endothelial cells (HUVECs) decreased as well as vascular endothelial growth factor expression following vorinostat and EGCG combined treatment. These results indicate that the combination of vorinostat and EGCG had a synergistic effect on inhibiting tumor cell angiogenesis potential. We suggest that the combination of vorinostat and EGCG is a novel option for cholangiocarcinoma chemotherapy.
\end{abstract}

\section{Introduction}

Cholangiocarcinoma, which arises from biliary tract epithelia, is a rare malignancy associated with poor prognosis and high mortality [1-3]. The incidence rate of cholangiocarcinoma has increased worldwide [4-6]. However, the reason for the increase in cholangiocarcinoma remains unclear. Cholangiocarcinoma accounts for 3\% of all gastrointestinal cancers and for approximately $15 \%$ of liver cancers worldwide [4-7]. Cholangiocarcinoma progresses insidiously and is difficult to diagnose. Although surgical resection is a unique option for curative treatment of cholangiocarcinoma, patients diagnosed with cholangiocarcinoma are frequently identified in an advanced stage and cannot be practically considered for surgical resection. Palliative therapies such as chemotherapy, radiotherapy, displacement of drug-eluting stents, and photodynamic therapy can be considered to increase patient quality of life. Although cholangiocarcinoma scarcely respond to traditional chemotherapy $[8,9]$ and has a poor prognosis, chemotherapy remains a feasible treatment option for cholangiocarcinoma. Most chemotherapeutic agents such as cisplatin, oxaliplatin, and gemcitabine inhibit cancer cell proliferation. However, systemic treatment of cholangiocarcinoma with these kinds of anticancer agents is always unsuccessful. Thus, a novel treatment option is required, as progression of cholangiocarcinoma is dependent on invasion, metastasis, spreading, and proliferation $[1,10-$ 12].

Acetylation/deacetylation of histones plays an important role in the transcriptional regulation of cells, and histone deacetylase inhibitor (HDACi) has been investigated as a promising new class of cancer chemotherapeutic drugs [1315]. A number of HDACis are in clinical trials for various neoplastic diseases. HDACis modify gene transcription, by 
chromatin remodeling and by changes in the structure of proteins in transcription factor complexes $[15,16]$. Vorinostat (suberoylanilide hydroxamic acid) was the first HDACi approved by the US Food and Drug Administration for clinical use to treat advanced cutaneous $\mathrm{T}$ cell lymphoma. Vorinostat induces differentiation, growth arrest, and apoptosis of various tumor cells in culture $[14,15]$. Furthermore, vorinostat has antiangiogenic activity [17].

The beneficial effect of (-)-epigallocatechin-3-gallate (EGCG), which is a major polyphenolic constituent of green tea, has been reported for various kinds of cancer [18-23]. EGCG is a potent antioxidant and has an anti-proliferative effect against tumors without adverse harmful effects on normal cells $[21,24]$. EGCG regulates expression of VEGF, MMPs, PA, insulin-like growth factor, epidermal growth factor receptor, and cell cycle regulatory proteins and inhibits NF- $\kappa$ B, PI3-K/Akt, Ras/Raf/mitogen activated protein kinase, and activator protein 1 signaling pathways [18]. These biological activities produce meaningful chemopreventive effects against cancer. In particular, EGCG has antiinvasive and antimetastatic effects on cancer cells [25-27]. EGCG also modulates acetylation of the androgen receptor by antihistone acetyltransferase activity and then suppresses prostate cancer cell growth [28].

In this study, we investigated the combined synergistic effect of vorinostat and EGCG against HuCC-T1 human cholangiocarcinoma cells in vitro. A synergistic chemopreventive effect against human cholangiocarcinoma cells was expected because vorinostat and EGCG have anti-proliferative, antiangiogenic, and anti-invasive effects [14, 17, 19-28]. We studied the combined effect of vorinostat and EGCG for the apoptosis, cytotoxicity, invasion, and angiogenic potential of HuCC-T1 cells in vitro.

\section{Material and Methods}

2.1. Preparation of Materials and Reagents. EGCG was purchased from Sigma Chem. Co. (St. Louis, MO, USA) and was dissolved in DMSO $(20 \mathrm{mg} / \mathrm{mL})$ as a stock solution. Vorinostat was purchased from LC Lab Co. and was dissolved in DMSO (50 mg/mL). RPMI1640 media, fetal bovine serum (FBS), and all cell culture components were purchased from Life Technologies (Grand Island, NY, USA). All reagents used were extrapure grade.

2.2. Cell Cultures. HuCC-T1 cholangiocarcinoma cells were obtained from the Health Science Research Resources Bank (Osaka, Japan) and maintained with RPMI1640 medium supplemented with $10 \%$ heat-inactivated FBS and $1 \%$ penicillin/streptomycin at $37^{\circ} \mathrm{C}$ in a humidified atmosphere containing $5 \% \mathrm{CO}_{2}$.

\subsection{Trypan Blue Exclusion Assay (Inhibition of Cell Growth} and Cytotoxicity Assay). HuCC-T1 cells were seeded in 24well plates at a density of $3 \times 10^{4}$ and $3 \times 10^{5}$ cells per well for growth inhibition and cytotoxicity assay, respectively. And then, each well plate was incubated overnight in a $\mathrm{CO}_{2}$ incubator. Vorinostat and EGCG in DMSO were diluted with RPMI1640 media containing 10\% FBS for growth inhibition assay at various concentrations and then added to tumor cells in 24-well plates following a 24-, 48-, and 72-hours incubation. And cytotoxicity assay was measured using serum-free RPMI1640 media. The control was treated with $0.1 \%(\mathrm{v} / \mathrm{v})$ DMSO. The cells were trypsinized, harvested, and resuspended in PBS. Trypan blue was added, and then the number of cells was counted using the Countess Automated Cell Counter (Invitrogen, Carlsbad, CA, USA). Inhibition of tumor cells growth was determined as the percentage of treated cells versus control cells.

2.4. Annexin V/Propidium Iodide (PI) Binding Assay. HuCCT1 cells were seeded in 6 -well plates at a density of $1 \times$ $10^{6}$ cells per well and exposed to various concentrations of vorinostat and EGCG for 24 hours. The cells were harvested, washed with PBS, resuspended in $100 \mu$ l binding buffer, and stained with FITC-conjugated Annexin V for the apoptosis analysis and with PI for the necrosis analysis. These cells were analyzed by flow cytometry.

2.5. Protein Lysates and Western Blot Analysis. Cells were trypsinized and washed with cold PBS. The cells were collected by centrifugation and lysed in lysis buffer containing protease inhibitors (50 mM Tris, $150 \mathrm{mM} \mathrm{NaCl}, 1 \% \mathrm{NP}-40$, $0.5 \%$ deoxycholic acid, $0.1 \%$ sodium dodecyl sulfate, [SDS]) with phenylmethylsulfonyl fluoride and a protease inhibitor cocktail (Roche Diagnostics, Indianapolis, IN, USA). The cell suspension was cleared by centrifugation at $14,000 \times \mathrm{g}$ for $30 \mathrm{~min}$ at $4^{\circ} \mathrm{C}$, and then supernatant or cell lysates were collected. Protein concentration was determined using the BCA Protein Assay kit (Pierce, Rockford, IL, USA).

For Western blotting, $50 \mu \mathrm{g}$ protein was subjected to SDSpolyacrylamide gel electrophoresis (SDS-PAGE), transferred to a PVDF membrane, blocked with 5\% skim milk in TBS-T, then probed with an appropriate primary antibody followed by a secondary HRP-conjugated antibody. Proteins were detected by chemiluminescence. Blots were subsequently stripped and reprobed with anti- $\beta$-actin primary antibody followed by the appropriate secondary antibody and chemiluminescence detection as a loading control. Proteins were quantified by digital analyses of the protein bands using the ImageJ software program.

2.6. Gelatin Zymography. HuCC-T1 cells $\left(1 \times 10^{6}\right.$ cells/well $)$ were seeded in 6-well plates and exposed to various concentrations of vorinostat and EGCG for 24 hours. MMP activity in the conditioned medium was analyzed by substrategel electrophoresis using SDS-PAGE containing 10\% gelatin (gelatin zymography). Equal volumes of conditioned cell culture medium samples were mixed with Laemmli buffer under nonreducing conditions, loaded onto the gel and separated by electrophoresis. SDS was removed by soaking the gels three times for $30 \mathrm{~min}$ at room temperature in Triton buffer (2.5\% Triton X-100 in PBS) and the gels were incubated for 24 hours at $37^{\circ} \mathrm{C}$. The gels were stained with $0.1 \%$ Coomassie Brilliant Blue R-250 and destained until clear bands became evident. Quantitative results of the assays were obtained by densitometry. 
2.7. TUNEL Assay. Fragmented DNA indicating apoptosis of tumor cells was observed by TUNEL. Cells exposed to various combinations of vorinostat and EGCG for 24 hours were fixed in $4 \%$ paraformaldehyde in PBS and then stained with an in situ apoptosis detection kit (Millipore, Billerica, MA, USA) according to manufacturer's protocol. Briefly, slides were incubated with $3 \% \mathrm{H}_{2} \mathrm{O}_{2}$ for 10 minutes at room temperature to block endogenous peroxidase activity and then with the TdT enzyme in a humidified chamber at $37^{\circ} \mathrm{C}$ for 1 hour. Subsequently, the slides were incubated with blocking reagent in a humidified chamber at $37^{\circ} \mathrm{C}$ for 30 minutes and then incubated with anti-DIGOiygenin-biotin $(1: 100)$ at $37^{\circ} \mathrm{C}$ for 30 minutes. After the streptavidin-biotin complex (SABC, $1: 100$ ) was applied to the slides at $37^{\circ} \mathrm{C}$ for 30 minutes, immunoreactivity was visualized with the Alexa488 secondary antibody. The negative control used distilled water in place of the TdT solution. The presence of clear nuclear staining was indicative of apoptotic cells. At least 500 cell nuclei were examined in the most evenly and distinctly labeled areas. The number of TUNEL-positive tumor cell nuclei was counted, and the apoptotic index was the percentage of apoptotic cells in the tumor.

2.8. Cell Invasion Assay. The invasion assay was performed as reported previously [12]. Transwell chambers in 24-well plates were employed to measure invasive potential of tumor cells. The upper chamber was coated with $20 \mu \mathrm{L}$ Matrigel (1 mg/mL; BD Bioscience, San Jose, CA, USA). HuCC-T1 cells were seeded on the upper parts of the Transwell chamber at $2 \times 10^{4}$ cells in $100 \mu \mathrm{L}$ serum-free medium with or without vorinostat and EGCG. The chamber was placed into the 24well plate, which contained $600 \mu \mathrm{L}$ of RPMI1640 containing $10 \%$ FBS. These cultures were incubated for 24 hours at $37^{\circ} \mathrm{C}$ in a $\mathrm{CO}_{2}$ incubator. Cells on the upper surface of the membrane were fixed with methanol, stained with hematoxylin and eosin, and photographed at the end of the incubation. Cells from various areas of the lower surface of the membrane were counted using a computerized video image analyzing system. Each assay was performed in triplicate, and mean \pm standard deviation is presented.

2.9. Wound Healing Assay. A wound healing assay of HuCCT1 CC cells was performed using a wound healing assay kit containing ibidi Culture-Inserts (ibidi GmbH, Planegg/ Martinsried Germany) as reported previously [12]. Aliquots containing $5 \times 10^{5}$ cells in RPMI1640 supplemented with $10 \%$ FBS were seeded on 6-well plates and the cells were exposed to vorinostat and EGCG at $37^{\circ} \mathrm{C}$ and $5 \% \mathrm{CO}_{2}$ for 24 hours. The cells were then washed twice with PBS and harvested by trypsinization. Then, $5 \times 10^{4}$ cells in serum free RPMI1640 were seeded into the culture inserts following a 24-hour incubation. The wound healing and migrated cell zone was observed using light microscopy. Serum-free medium was used to avoid proliferation-dependent migration of tumor cells.

2.10. Antiangiogenesis Assay. An angiogenesis assay was performed as reported by Okabe et al. [29]. When HuCC-T1 cells filled dishes to $70-80 \%$ confluency, the medium was replaced with serum-free RPMI1640 and then cells were treated with vorinostat and EGCG for 24 hours. The media were centrifuged at $1000 \mathrm{rpm}$ for $5 \mathrm{~min}$ and the supernatant was used as the medium. Protein content of the medium was determined with a BCA Assay kit (Pierce) and aliquots were stored at $-80^{\circ} \mathrm{C}$ until use. HUVECs $\left(1 \times 10^{4}\right.$ cells/well $)$ were suspended in a mixture of Medium/EGM-2 medium (1:1) with $0.5 \%$ FBS and then seeded on $50 \mu \mathrm{L}$ of Matrigel in 96-well plates. These plates were incubated for 12 hours, and cell morphology was examined in each well. The total capillary tube length and branching points were examined in three random view fields per well, and the average values were calculated.

2.11. Statistical Analysis. Statistical analyses of the data between treated and untreated cells were performed using Student's $t$-test. A $P$ value $<0.05$ was considered significant. * represents statistical significance compared to the vehicletreated control.

\section{Results}

3.1. Inhibition of Cell Growth and Cytotoxic Effect of Vorinostat and EGCG on the HuCC-T1 Cell Line. Tumor cell proliferation and viability were determined using the Trypan blue dye exclusion assay to identify the synergistic effect of the combination of vorinostat and EGCG. The $\mathrm{IC}_{50}$ values of the vorinostat and EGCG treatment alone were 2.4609 and $0.2148 \mu \mathrm{g} / \mathrm{mL}$, respectively. One and $5 \mu \mathrm{g} / \mathrm{mL}$ vorinostat were used to test the combined effect with EGCG. As shown in Figure 1(e), synergistic inhibition of growth (85\%) was observed with the vorinostat and EGCG combination, whereas each agent alone resulted in $70 \%$ inhibition for EGCG and 75\% for vorinostat. A synergistic cellular cytotoxicity effect was also observed with the combined vorinostat and EGCG treatment, as shown in Figure 1(f). EGCG did not have a cytotoxic effect because $\geq 60 \%$ and $78 \%$ cells survived at 10 and $5 \mu \mathrm{g} / \mathrm{mL}$ EGCG, respectively. As shown in Figure 1(d), the vorinostat $\mathrm{IC}_{50}$ was $1.2305 \mu \mathrm{g} / \mathrm{mL}$. Figure $1(\mathrm{f})$ showed that significant toxicity of up to $52 \%$ with vorinostat and $23 \%$ with EGCG was observed. The vorinostat and EGCG combination caused significantly greater cholangiocarcinoma toxicity (65\%) (Figure 1(f)).

\subsection{Apoptosis and Necrosis of HuCC-T1 Cholangiocarcinoma} Cells following Vorinostat and EGCG Treatment. Figure 2 shows the apoptosis and necrosis analysis of HuCC-T1 cholangiocarcinoma cells in response to vorinostat and EGCG. As shown in Figure 2(a), both of vorinostat and EGCG induced tumor cell apoptosis rather than necrosis. In particular, the vorinostat and EGCG combination significantly enhanced apoptosis in HuCC-T1 cells. The apoptosis index of the vorinostat and EGCG combination was approximately three fold higher than that when vorinostat or EGCG was treated alone. These results indicate that the combination of vorinostat and EGCG induced synergistic anticancer effects on tumor cells. Furthermore, the TUNEL assay showed that the vorinostat and EGCG combination had a synergistic anticancer effect. Fluorescence intensity in the combined vorinostat and EGCG treatment was obviously higher than 


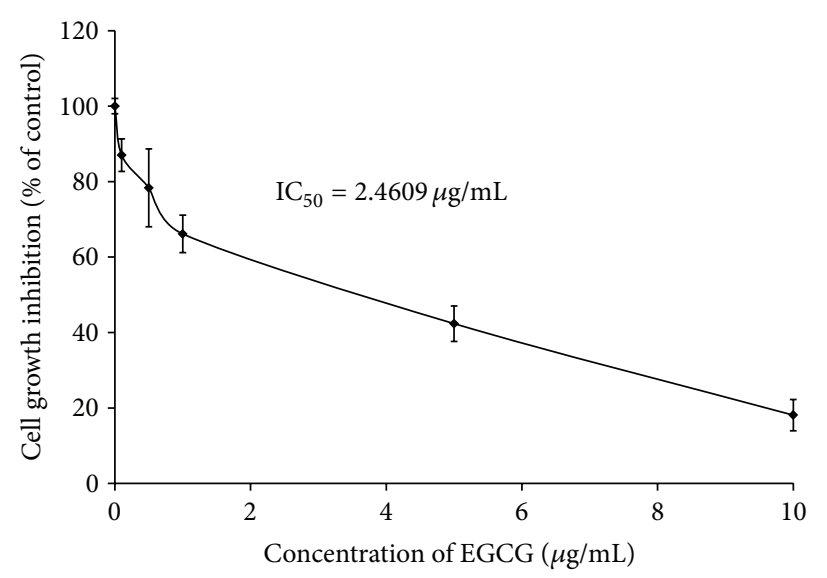

(a)

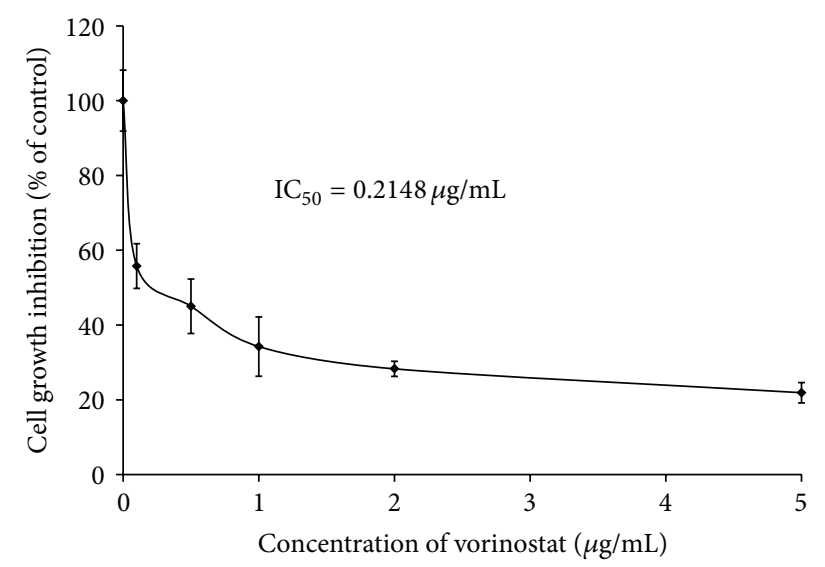

(c)

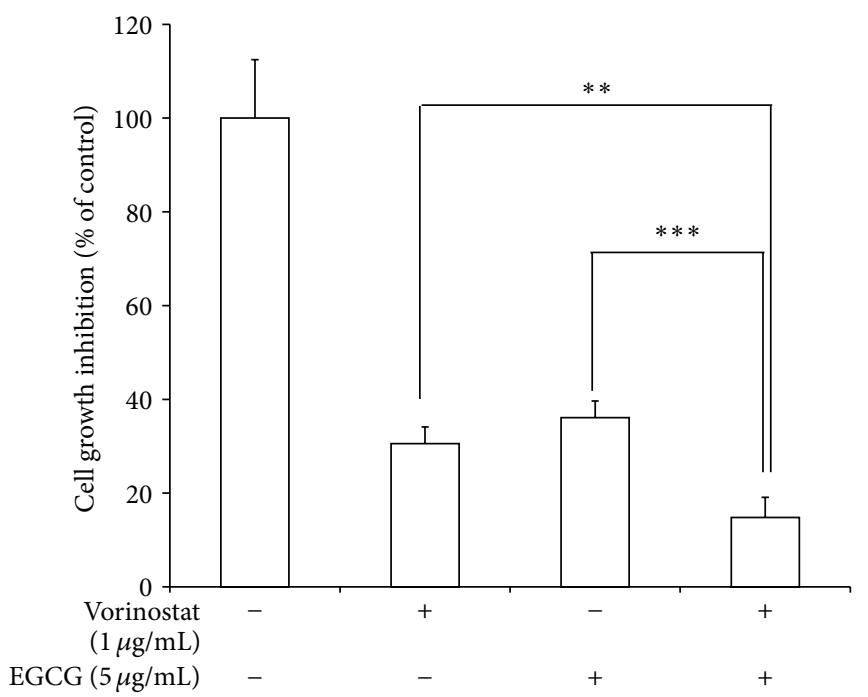

(e)

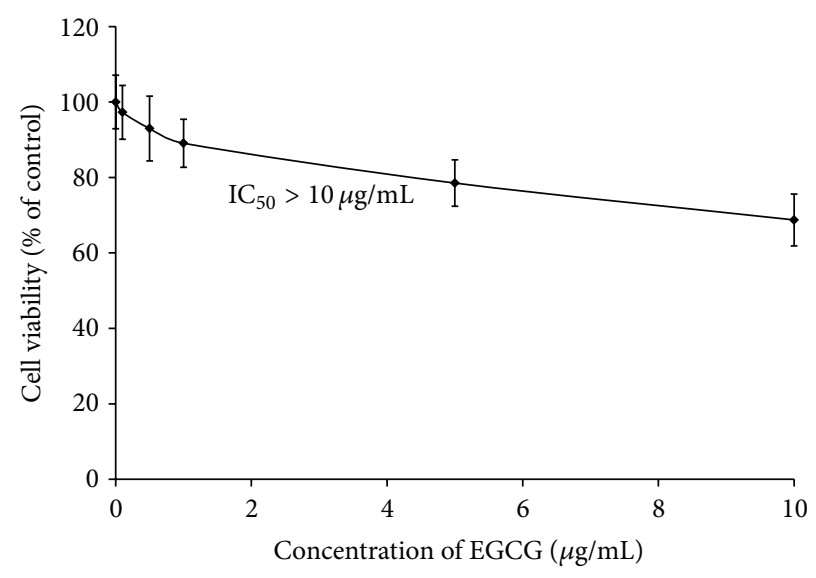

(b)

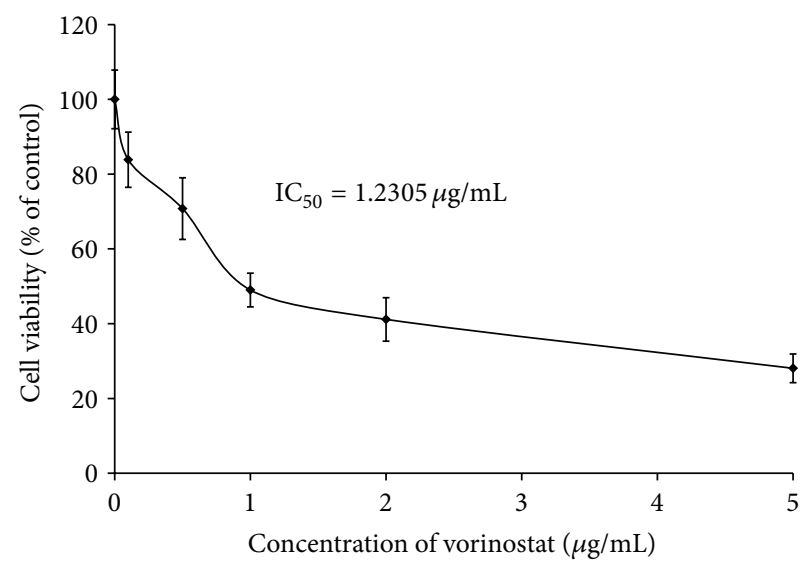

(d)

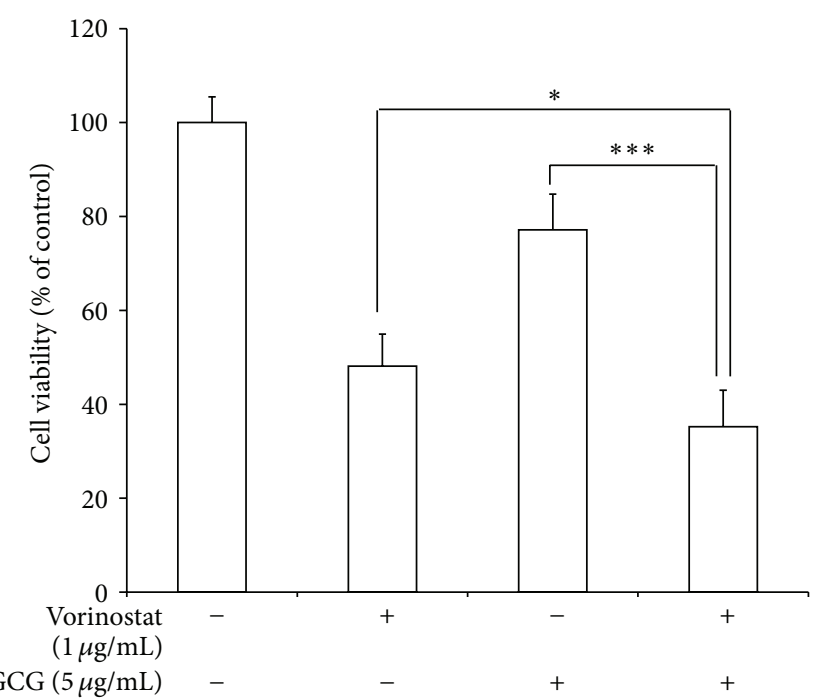

(f)

FIGURE 1: The synergistic anticancer effects of vorinostat and EGCG were measured by cytotoxic and growth inhibition responses. A $3 \times 10^{4}$ aliquot of cells for the cell cytotoxicity assay and $3 \times 10^{3}$ cells for growth inhibition assay were seeded in 96-well plates. RPMI1640 media supplemented 10\% FBS was used to asses tumor cell growth inhibition, whereas serum-free media were used for cell cytotoxicity assay. Single agent treatment: (a) and (b), EGCG; (c) and (d), vorinostat. Combined vorinostat and EGCG: (e) and (f). Growth inhibition: (a), (c), and (e). Cell cytotoxicity: (b), (d), and (f). ${ }^{*} P<0.05 ;{ }^{* *} P<0.01$; ${ }^{* * *} P<0.001$. 

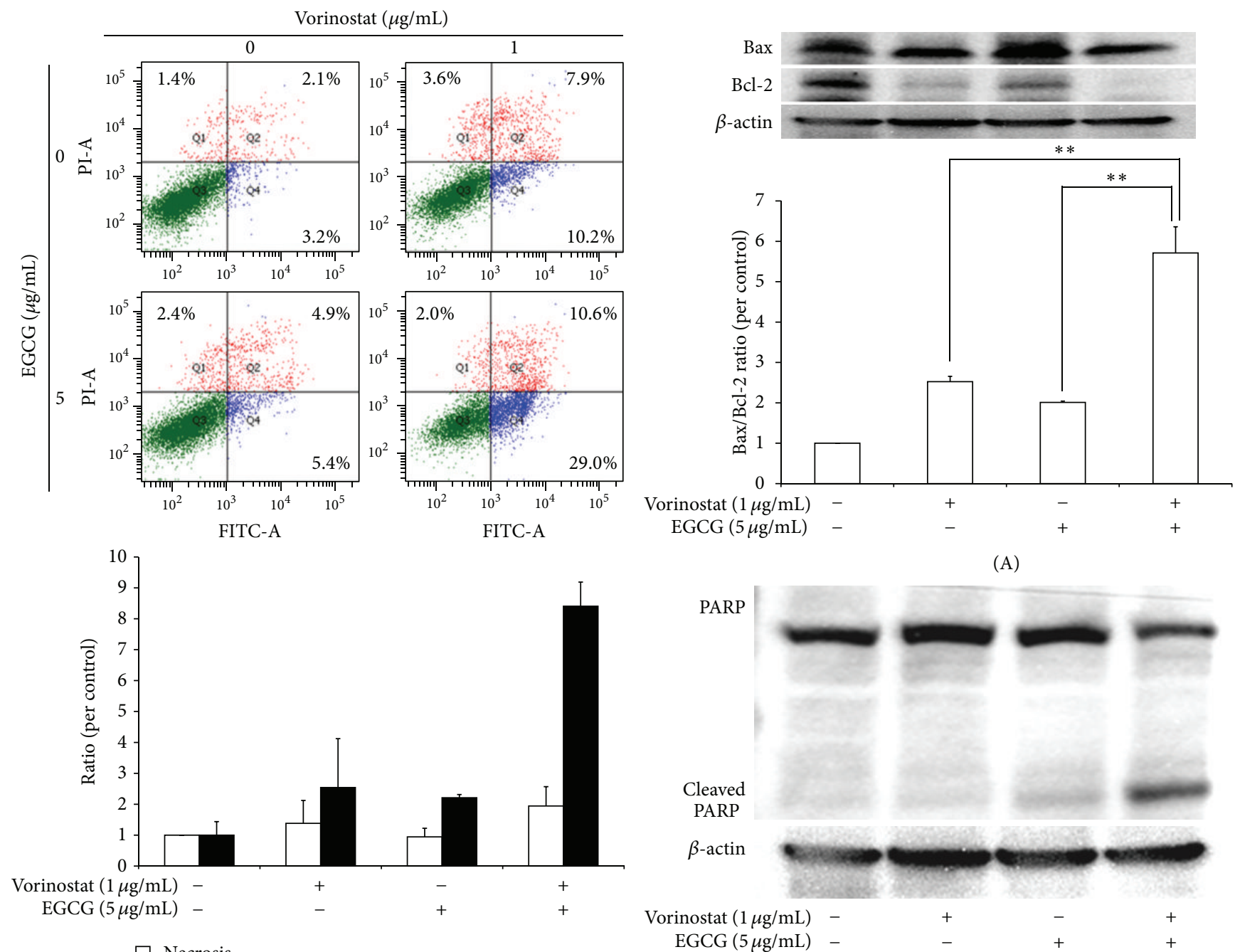

\section{$\square$ Necrosis}

- Apoptosis EGCG $(5 \mu \mathrm{g} / \mathrm{mL})$

(a)

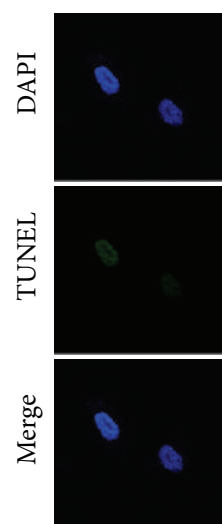

Vorinostat $(1 \mu \mathrm{g} / \mathrm{mL}) \quad-$ EGCG $(5 \mu \mathrm{g} / \mathrm{mL}) \quad-$

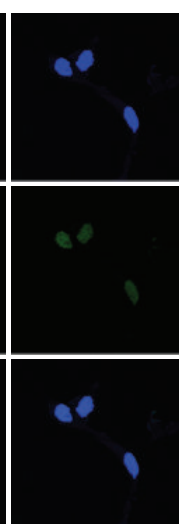

$+$

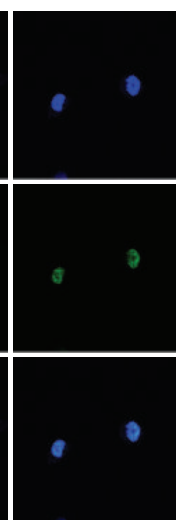

$-$

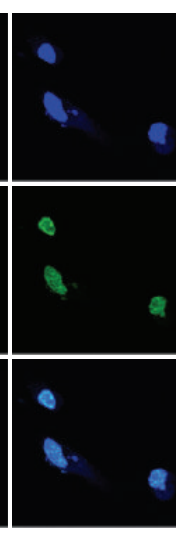

$+$

(b)

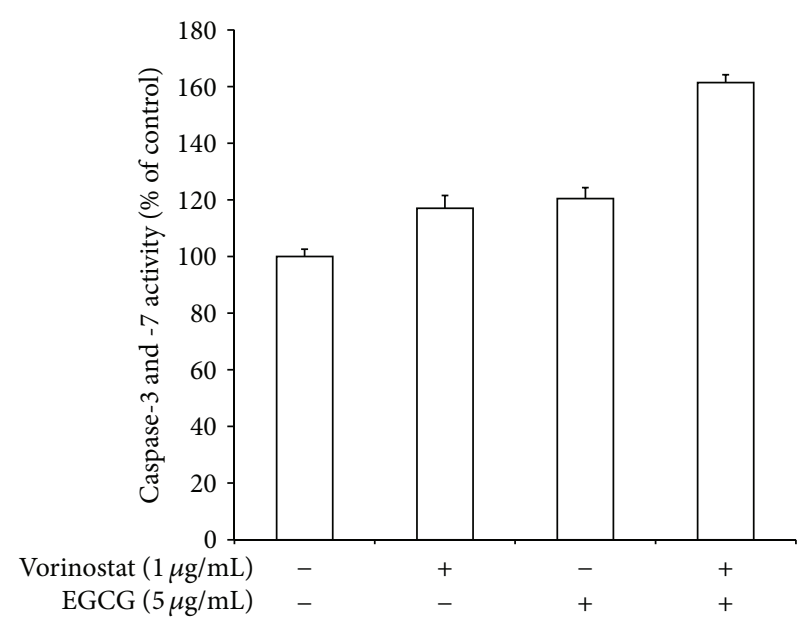

(C)

(c)

FIGURE 2: The synergistic effect of the combination of vorinostat and EGCG was assessed on apoptosis and necrosis of HuCC-T1 cholangiocarcinoma cells. (a) Flow cytometric analysis of tumor cells. FITC-Annexin V and propium iodide (PI) were used for apoptosis and necrosis analysis of tumor cells, respectively. (b) TUNEL assay. (c) Western blot assay: (A), Bax/Bcl-2 expression; (B), PARP expression; extent of caspase- 3 and-7 activity. ${ }^{* *} P<0.01$. 
that of the single treatments. The $\mathrm{Bax} / \mathrm{Bcl}-2$ expression ratio increased significantly twofold compared to individual treatment following treatment with the combination of vorinostat/EGCG. PARP, a protein involved in DNA repair and programmed cell death, also decreased following treatment with the vorinostat and EGCG combination, whereas the single treatments showed obvious increased protein intensities for both vorinostat and EGCG alone. Furthermore, caspase-3 and -7 activity of the combination was also higher than that of the single treatments.

These results indicate that the vorinostat and EGCG combination induced a synergistic anti-cancer effect against HuCC-T1 human cholangiocarcinoma cells.

3.3. Anti-Invasion, Antimigration, and Antiangiogenesis Effects. Figures 3 and 4 show invasion, migration, and angiogenesis behavior of HuCC-T1 human cholangiocarcinoma cells with single and combined treatment. The tumor cell invasive potential was assessed by Matrigel invasion assay. As shown in Figure 3(a), vorinostat/EGCG combinations were markedly inhibited invasion of tumor cells compared to single treatment of vorinostat or EGCG; that is, tumor cell invasive capacity was inhibited $13 \%$ versus vorinostat single treatment and $45 \%$ versus EGCG single treatment. Furthermore, MMP-2 and -9 , which play a crucial role in basement membrane degradation and tumor cell invasion, also decreased following the combined vorinostat and EGCG treatment and the expression level of MMP-2 or -9 was significantly lower at drug combination than single treatment. The wound healing assay was employed to study the effect of the combined vorinostat and EGCG treatment on tumor cell migration potential. Migration potential of HuCC-T1 cholangiocarcinoma cells was also obviously inhibited by the combined vorinostat and EGCG treatment compared to single treatment (Figure 3(c)).

Figure 4 shows the anti-angiogenesis effect of the vorinostat and EGCG combination in HUVECs. As shown in Figure 4(a), treated media from tumor cell cultures treated with the combination of vorinostat and EGCG or the agents alone showed the highest capacity to inhibit HUVEC tube and junction formation. Furthermore, VEGF was suppressed by the combined treatment (Figure 4(b)).

These results indicated that a combination of vorinostat and EGCG has synergistic effects to suppress invasion and migration of HuCC-T1 cells and suppress the angiogenesis potential of HUVECs.

\section{Discussion}

Conventional chemotherapeutic agents have very low therapeutic efficacy for cholangiocarcinoma [1, 10-12]. Although palliative therapies such as chemotherapy, radiotherapy, drug-eluting stents, and photodynamic therapy have beneficial effects as cholangiocarcinoma treatments, they are incomplete. Thus, a novel treatment option is required to suppress proliferation, invasion, angiogenesis, and migration of cholangiocarcinoma at the bile duct. We focused on the possibility of inhibiting proliferation, migration, invasion, and angiogenesis of cholangiocarcinoma using vorinostat and EGCG.

Vorinostat is an HDACi. Because histone acetylation facilitates loose chromatin structure and activates transcriptional potential, HDACs act as a transcription repressors and accelerate chromatin condensation [15]. HDACis such as vorinostat modify DNA transcription through chromatin remodeling and change the protein structure of transcription factor complexes. Vorinostat induces apoptosis, differentiation, proliferation inhibition, invasion inhibition and suppresses tumor cell angiogenesis potential $[14,15,17$, 30]. Takada et al. [14] reported that vorinostat potentiates apoptosis and inhibits invasion of various tumor cells by suppressing NF- $\kappa$ B. As several genes including antiapoptotic, proliferative, and angiogenic products are regulated by NF$\kappa \mathrm{B}$, proliferation, invasion, and angiogenesis potential of tumor cells can be regulated or inhibited by vorinostat. Furthermore, vorinostat induces oxidative stress against various types of cancer cells $[13,15,31]$. Although cancer cells generate more ROS than those of normal cells, vorinostat induces further oxidative stress in tumor cells and enhances the suppression of cancer cell proliferation. In recent decades, the antitumor efficacy of vorinostat against tumor patients has been well demonstrated in clinical trials [32-36]. Kelly et al. reported that vorinostat with daily intravenous (i.v.) administration has antitumor efficacy against solid and hematological tumors and inhibits the biological target in vivo [32]. Furthermore, oral administration of vorinostat was also demonstrated to have antitumor activity in patients with advanced cancer [33,34]. Clinical trials of vorinostat have been also performed against metastatic breast cancer and recurrent or metastatic head and neck cancer $[35,36]$. Especially, vorinostat is also known to inhibit independently proliferation, invasion, and migration of glioma cells at $2 \mathrm{D}$ and $3 \mathrm{D}$ culture in vitro [37]. The potential of vorinostat against tumor patients with recurrent glioblastoma multiforme was also reported in clinical trials [38]. In our results, we also observed that vorinostat alone and vorinostat/EGCG combination induce inhibition of MMP-2/9 and invasionof HuCC-T1 cells (Figure 3 and Supplementary Figure 4 available online at doi: http://dx.doi.org/10.1155/2013/185158).

The principal merit of EGCG is to induce tumor cell apoptosis without adverse effect in normal cells. EGCG not only has a beneficial effect on tumor cell apoptosis but also inhibits tumor cell invasion and metastasis [18-27]. EGCG inhibits growth of prostate and breast tumors in nude mice $[19,20]$. EGCG inhibits invasion and metastasis of tumor cells by regulating the activity of enzymes such as MMPs [25$27,39]$. EGCG inhibits migration, invasion, and spreading of melanoma cells dose dependently [39]. Furthermore, EGCG inhibits tyrosine phosphorylation of focal adhesion kinase and MMP-9 activity [40, 41]. As shown in Figure 3, our study showed that EGCG inhibited invasion and migration of HuCC-T1 human cholangiocarcinoma cells and suppressed MMP-9 activity.

This is the first report on the synergistic effect of vorinostat and EGCG for inhibiting proliferation, invasion, migration, and angiogenesis of human cholangiocarcinoma cells. In fact, vorinostat and EGCG have been frequently used 

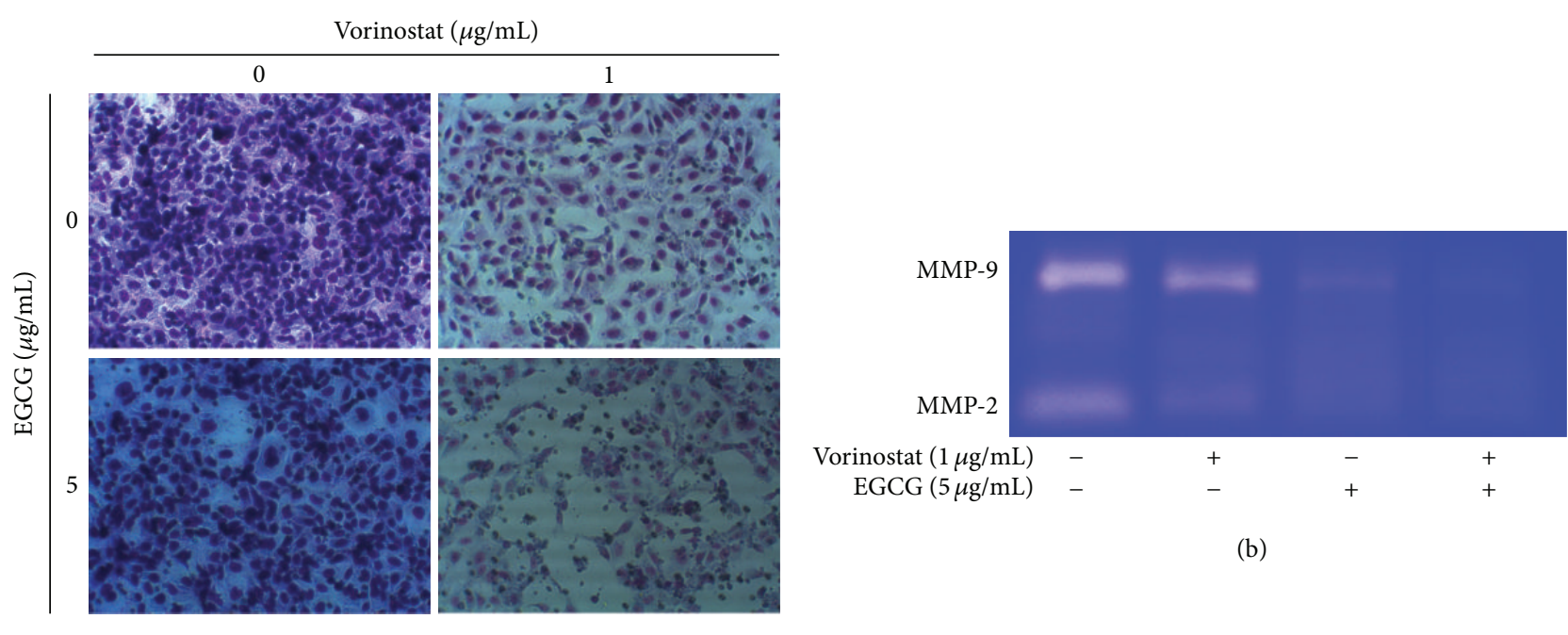

(b)

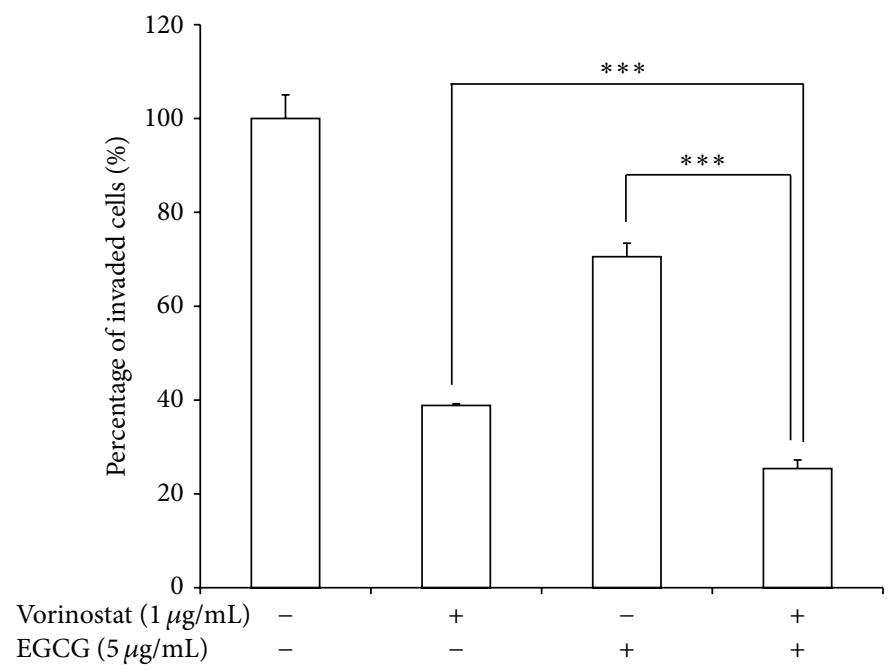

(a)

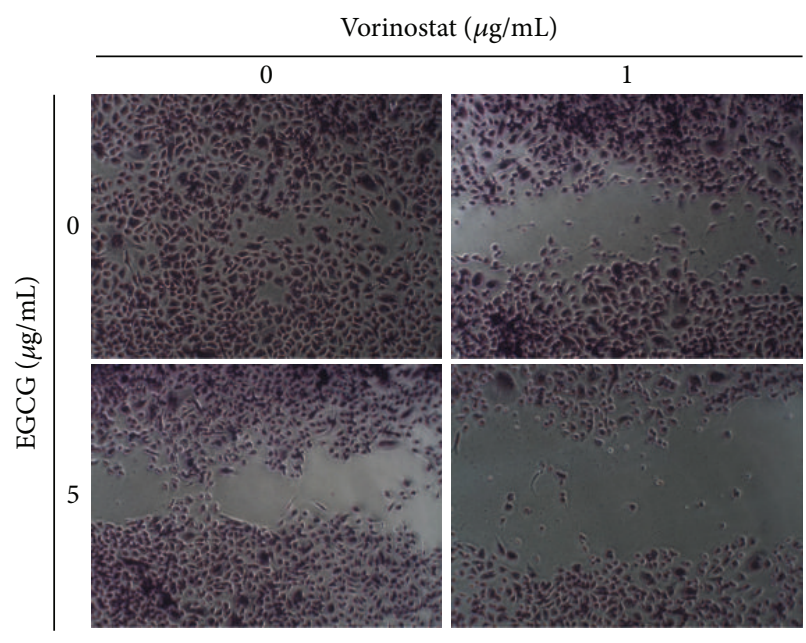

(c)

FIGURE 3: The combined effect of vorinostat and EGCG was assessed on invasion and migration capacity of HuCC-T1 cholangiocarcinoma cells. (a) Matrigel invasion assay. (b) Gelatin zymography: MMP-2 and -9 expression. (c) Wound healing assay for tumor cell migration. ${ }^{* * *} P$ $<0.001$.

in combination rather than as single agents [14, 31, 40-42]. Vorinostat has synergistic effects against various cancer cells in combinations with decitabine, tumor necrosis factor, cisplatin, 5-fluorouracil, doxorubicin, and paclitaxel [14, 31]. Nihal et al. [41] reported that the vorinostat and EGCG combination synergistically inhibits growth of melanoma cells by enhancing apoptosis and activating p21, p27, and caspases. The vorinostat and EGCG combination also downregulated the antiapoptotic protein $\mathrm{Bcl}-2$ and upregulated proapoptotic protein Bax (Figure 2(c)). Lang et al. reported that EGCG accelerates apoptosis in human cholangiocarcinoma cells when used in combination with gemcitabine. As shown in Figure 2, we also found that the vorinostat and EGCG combination enhanced apoptotic signals, increased the $\mathrm{Bax} / \mathrm{Bcl}-2$ ratio, and promoted cleaved PARP expression level. Furthermore, the synergistic effects of drug combination were maintained for 72 hours (Supplementary Figure 1). The synergistic effects of induced apoptosis (Figure 2) and modulation of the HuCC-T1 cholangiocarcinoma cell cycle were observed (Supplementary Figure 2). p53 expression also increased following the combined vorinostat and EGCG treatment (Supplementary Figure 3).

Vorinostat and EGCG have contrasting tumor cell oxidative stress behavior, as vorinostat induces ROS whereas EGCG is an antioxidant [31, 41]. EGCG is reported to complement vorinostat, as vorinostat activity decreased under high ROS levels in cell culture but markedly inhibits tumor cell growth at a low ROS level [43]. However, pretreatment with antioxidants reduces cellular ROS level and synergistically sensitizes tumor cell oxidative stress by vorinostat following higher tumor cell growth inhibition compared to that of a single vorinostat treatment. In our study, vorinostat alone induced increased cellular ROS but ROS level decreased significantly both in the EGCG only and vorinostat and EGCG combined treatments (Supplementary Figure 5). EGCG treatment to HuCC-T1 cells was resulted in a decrease in cellular 

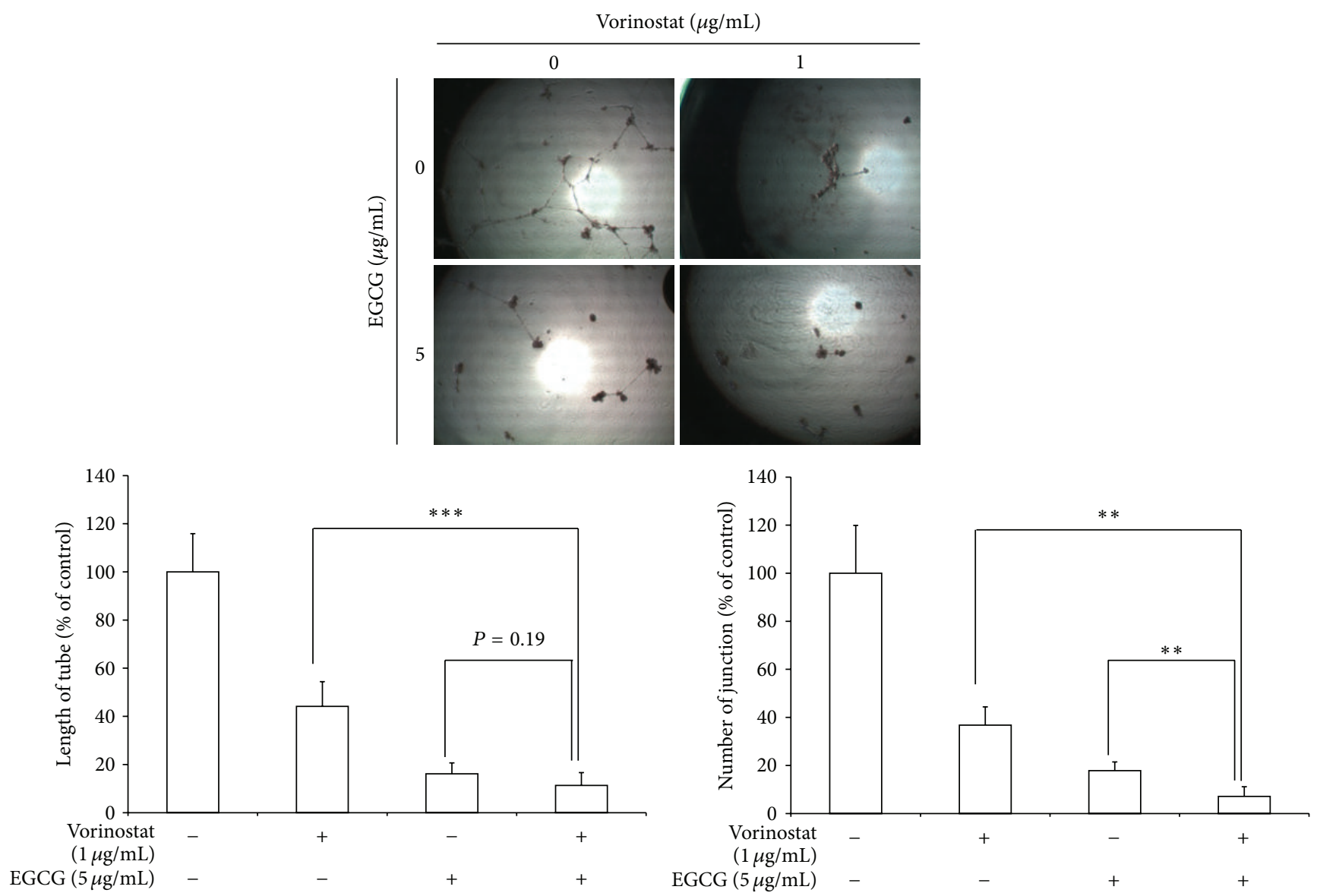

(a)

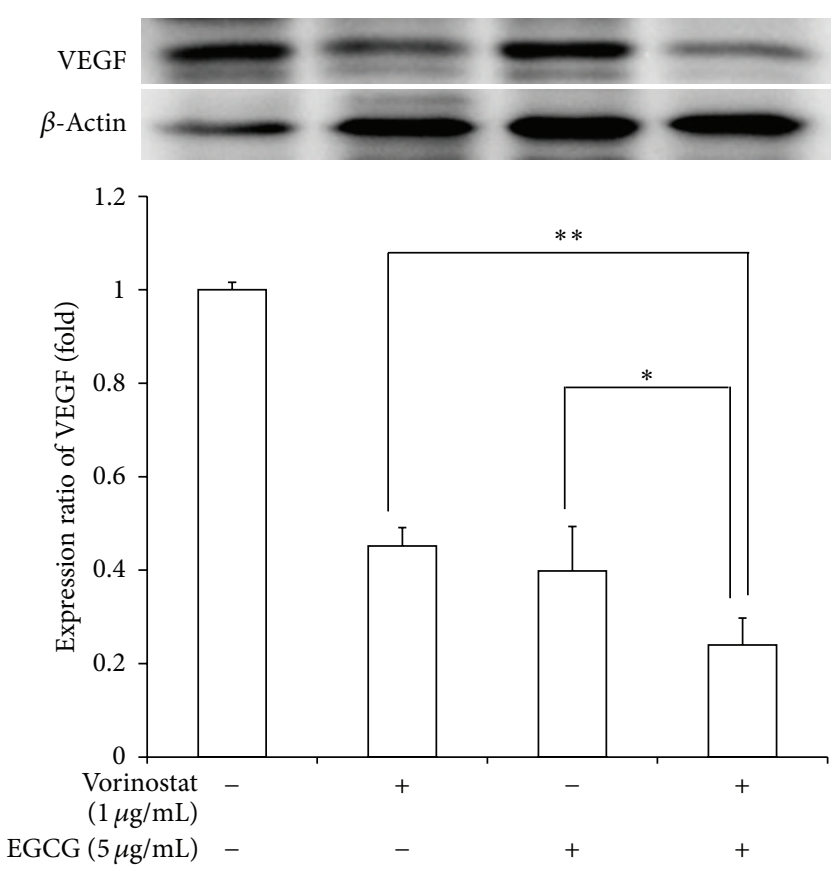

(b)

FIGURE 4: The combined effect of vorinostat and EGCG was assessed on angiogenic activity of tumor cells. HUVECs were used for the angiogenesis assay. Treated media from tumor cell cultured with the combination of vorinostat and EGCG were added to HUVECs. (a) The length of tubes and the numbers of junction were counted and compared with those of the control. (b) Western blot assay: VEGF expression and ratio. ${ }^{*} P<0.05 ;{ }^{* *} P<0.01 ;{ }^{* * *} P<0.001$. 
ROS level compared to nontreated cells. The tendency for the ROS decrease was not reversibly changed by combined treatment of vorinostat and EGCG (Supplementary Figure 5), indicating that antioxidants effect of EGCG might be dominant in the molecular mechanism of oxidative stress in the cells. Basu et al. reported that pretreatment with antioxidants sensitizes growth inhibitory effect of vorinostat against oxidatively stressed human cancer cells [43]. They argued that vorinostat treatment markedly inhibits growth of LNCaP cells when the cells are at a low ROS and, however, potency of vorinostat was significantly decreased against the same cell line at high ROS level. Pretreatment of vitamin E as an antioxidant reduced cellular ROS level and then synergistically sensitized oxidatively stressed LNCaP cells [43]. They also demonstrated that the potency of vorinostat against cancer cells could be improved by combination with antioxidants. Furthermore, EGCG is known to stimulate cancerspecific induction of ROS and to induce preferential death of cancer cells [24]. Nihal et al., also reported that combination of EGCG and vorinostat results in significant inhibition of cell proliferation and increase in apoptotic signals [41]. Li et al. observed that estrogen receptor- $\alpha(\mathrm{ER} \alpha)$ expression in $\mathrm{ER} \alpha$ negative MDA-MB-231 breast cancer cells was synergistically reactivated by combination of EGCG and trichostatin A (TSA) [44]. They also found that EGCG/TSA combination was effective to inhibit HDACs activity and cell viability. Furthermore, they observed that EGCG alone significantly inhibited binding of HDACl. Many investigations also reported that EGCG affects the activity of HDAC and histone acetyltransferase (HAT) [28, 41, 44-46]. Choi et al. identified EGCG as a potent HAT inhibitor (HATi) [45]. They observed that EGCG has global specificity for the majority of HAT enzymes but with negligible effect on the HDAC activity. Other reports showed that EGCG induced Raf kinase inhibitor protein (RKIP) upregulation via the inhibition of histone deacetylase (HDAC) activity [46].

Angiogenesis potential of HUVECs and VEGF expression in HuCC-Tl cholangiocarcinoma cells also decreased with the combined treatment of vorinostat and EGCG (Figure 4). HDACis such as vorinostat inhibit VEGF-induced expression of VEGF receptors in endothelial cells and then inhibit angiogenesis [15].

Our results demonstrated the synergistic effect of a combination of vorinostat and EGCG on suppressing proliferation, invasion, migration, angiogenesis, and induction of apoptosis. We suggest that vorinostat and EGCG together are a novel treatment option for CCA chemotherapy.

\section{Abbreviations}

PARP: $\quad$ Poly (ADP-ribose) polymerase

MMP: $\quad$ Matrix metalloproteinase

EGCG: (-)-Epigallocatechin-3-gallate

HUVECs: Human umbilical vein endothelial cells

HDACi: Histone deacetylase inhibitor

VEGF: Vascular endothelial growth factor

NF- $\kappa$ B: $\quad$ Nuclear factor-kappa B
TUNEL: Terminal deoxynucleotidyl transferase dUTP nick end labeling

ROS: Reactive oxygen species.

\section{Acknowledgments}

This study was supported by a grant from the Korean Healthcare Technology R\&D Project, Ministry of Health and Welfare, Republic of Korea (Project No. A091047).

\section{References}

[1] J. H. Lim, "Cholangiocarcinoma: morphologic classification according to growth pattern and imaging findings," American Journal of Roentgenology, vol. 181, no. 3, pp. 819-827, 2003.

[2] D. S. Sandhu and L. R. Roberts, "Diagnosis and management of cholangiocarcinoma," Current Gastroenterology Reports, vol. 48, pp. 43-52, 2008.

[3] P. Singh and T. Patel, "Advances in the diagnosis, evaluation and management of cholangiocarcinoma," Current Opinion in Gastroenterology, vol. 22, no. 3, pp. 294-299, 2006.

[4] S. A. Khan, S. D. Taylor-Robinson, M. B. Toledano, A. Beck, P. Elliott, and H. C. Thomas, "Changing international trends in mortality rates for liver, biliary and pancreatic tumours," Journal of Hepatology, vol. 37, no. 6, pp. 806-813, 2002.

[5] T. Patel, "Increasing incidence and mortality of primary intrahepatic cholangiocarcinoma in the United States," Hepatology, vol. 33, no. 6, pp. 1353-1357, 2001.

[6] S. A. Khan, H. C. Thomas, B. R. Davidson, and S. D. TaylorRobinson, "Cholangiocarcinoma," Lancet, vol. 366, no. 9493, pp. 1303-1314, 2005.

[7] P. Sriwanitchrak, V. Viyanant, W. Chaijaroenkul et al., "Proteomics analysis and evaluation of biomarkers for detection of cholangiocarcinoma," Asian Pacific Journal of Cancer Prevention, vol. 12, no. 6, pp. 1503-1510, 2011.

[8] A. E. Sirica, "Cholangiocarcinoma: molecular targeting strategies for chemoprevention and therapy," Hepatology, vol. 41, no. 1, pp. 5-15, 2005.

[9] B. R. A. Blechacz and G. J. Gores, "Cholangiocarcinoma," Clinics in Liver Disease, vol. 12, no. 1, pp. 131-150, 2008.

[10] M. R. Bhuiya, Y. Nimura, J. Kamiya et al., "Clinicopathologic studies on perineural invasion of bile duct carcinoma," Annals of Surgery, vol. 215, no. 4, pp. 344-349, 1992.

[11] F. Z. Shen, B. Y. Zhang, Y. J. Feng et al., "Current research in perineural invasion of cholangiocarcinoma," Journal of Experimental \& Clinical Cancer Research, vol. 29, p. 24, 2010.

[12] K.-D. Chung, Y.-I. Jeong, C.-W. Chung, D. H. Kim, and D. H. Kang, "Anti-tumor activity of all-trans retinoic acid-incorporated glycol chitosan nanoparticles against HuCC-T1 human cholangiocarcinoma cells," International Journal of Pharmaceutics, vol. 422, no. 1-2, pp. 454-461, 2012

[13] V. M. Richon, "Cancer biology: mechanism of antitumour action of vorinostat (suberoylanilide hydroxamic acid), a novel histone deacetylase inhibitor," British Journal of Cancer, vol. 95, no. 1, pp. S2-S6, 2006.

[14] Y. Takada, A. Gillenwater, H. Ichikawa, and B. B. Aggarwal, "Suberoylanilide hydroxamic acid potentiates apoptosis, inhibits invasion, and abolishes osteoclastogenesis by suppressing nuclear factor- $\kappa \mathrm{B}$ activation," Journal of Biological Chemistry, vol. 281, no. 9, pp. 5612-5622, 2006. 
[15] W. S. Xu, R. B. Parmigiani, and P. A. Marks, "Histone deacetylase inhibitors: molecular mechanisms of action," Oncogene, vol. 26, no. 37, pp. 5541-5552, 2007.

[16] C.-Y. Gui, L. Ngo, W. S. Xu, V. M. Richon, and P. A. Marks, "Histone deacetylase (HDAC) inhibitor activation of p21WAF1 involves changes in promoter-associated proteins, including HDAC1," Proceedings of the National Academy of Sciences of the United States of America, vol. 101, no. 5, pp. 1241-1246, 2004.

[17] C. F. Deroanne, K. Bonjean, S. Servotte et al., "Histone deacetylases inhibitors as anti-angiogenic agents altering vascular endothelial growth factor signaling," Oncogene, vol. 21, no. 3, pp. 427-436, 2002.

[18] S. Shankar, S. Ganapathy, and R. K. Srivastava, "Green tea polyphenols: biology and therapeutic implications in cancer," Frontiers in Bioscience, vol. 12, no. 13, pp. 4881-4899, 2007.

[19] S. Liao, Y. Umekita, J. Guo, J. M. Kokontis, and R. A. Hiipakka, "Growth inhibition and regression of human prostate and breast tumors in athymic mice by tea epigallocatechin gallate," Cancer Letters, vol. 96, no. 2, pp. 239-243, 1995.

[20] R. L. Thangapazham, A. K. Singh, A. Sharma, J. Warren, J. P. Gaddipati, and R. K. Maheshwari, "Green tea polyphenols and its constituent epigallocatechin gallate inhibits proliferation of human breast cancer cells in vitro and in vivo," Cancer Letters, vol. 245, no. 1-2, pp. 232-241, 2007.

[21] A. Das, N. L. Banik, and S. K. Ray, "Flavonoids activated caspases for apoptosis in human glioblastoma T98G and U87MG cells but not in human normal astrocytes," Cancer, vol. 116, no. 1, pp. 164-176, 2010.

[22] Y. Qiao, J. Cao, L. Xie, and X. Shi, "Cell growth inhibition and gene expression regulation by (-)-epigallocatechin-3-gallate in human cervical cancer cells," Archives of Pharmacal Research, vol. 32, no. 9, pp. 1309-1315, 2009.

[23] B. J. Philips, C. H. Coyle, S. N. Morrisroe, M. B. Chancellor, and N. Yoshimura, "Induction of apoptosis in human bladder cancer cells by green tea catechins," Biomedical Research, vol. 30, no. 4, pp. 207-215, 2009.

[24] N. Y. Min, J.-H. Kim, J.-H. Choi et al., "Selective death of cancer cells by preferential induction of reactive oxygen species in response to (-)-epigallocatechin-3-gallate," Biochemical and Biophysical Research Communications, vol. 421, no. 1, pp. 91-97, 2012.

[25] L. Sartor, E. Pezzato, M. Donà et al., "Prostate carcinoma and green tea: (-)Epigallocatechin-3-gallate inhibits inflammationtriggered MMP-2 activation and invasion in murine tramp model," International Journal of Cancer, vol. 112, no. 5, pp. 823829, 2004.

[26] R. Benelli, R. Venè, D. Bisacchi, S. Garbisa, and A. Albini, "Anti-invasive effects of green tea polyphenol epigallocatechin3-gallate (EGCG), A natural inhibitor of metallo and serine proteases," Biological Chemistry, vol. 383, no. 1, pp. 101-105, 2002.

[27] M. S. Baliga, S. Meleth, and S. K. Katiyar, "Growth inhibitory and antimetastatic effect of green tea polyphenols on metastasis-specific mouse mammary carcinoma $4 \mathrm{~T} 1$ cells in vitro and in vivo systems," Clinical Cancer Research, vol. 11, no. 5, pp. 19181927, 2005.

[28] Y. H. Lee, J. Kwak, H. K. Choi et al., "EGCG suppresses prostate cancer cell growth modulating acetylation of androgen receptor by anti-histone acetyltransferase activity," International Journal of Molecular Medicine, vol. 30, pp. 69-74, 2012.
[29] H. Okabe, T. Beppu, H. Hayashi et al., "Hepatic stellate cells accelerate the malignant behavior of cholangiocarcinoma cells," Annals of Surgical Oncology, vol. 18, no. 4, pp. 1175-1184, 2011.

[30] J. Chandra, C. P. Miller, M. M. Singh, N. Rivera-Del Valle, and C. A. Manton, "Therapeutic strategies to enhance the anticancer efficacy of histone deacetylase inhibitors," Journal of Biomedicine and Biotechnology, vol. 2011, Article ID 514261, 17 pages, 2011.

[31] B. Brodská and A. Holoubek, "Generation of reactive oxygen species during apoptosis induced by DNA-damaging agents and/or histone deacetylase inhibitors," Oxidative Medicine and Cellular Longevity, vol. 2011, Article ID 253529, 7 pages, 2011.

[32] W. K. Kelly, V. M. Richon, O. O’Connor et al., "Phase I clinical trial of histone deacetylase inhibitor: suberoylanilide hydroxamic acid administered intravenously," Clinical Cancer Research, vol. 9, no. 10 I, pp. 3578-3588, 2003.

[33] W. K. Kelly, O. A. O'Connor, L. M. Krug et al., "Phase I study of an oral histone deacetylase inhibitor, suberoylanilide hydroxamic acid, in patients with advanced cancer," Journal of Clinical Oncology, vol. 23, no. 17, pp. 3923-3931, 2005.

[34] O. A. O'Connor, M. L. Heaney, L. Schwartz et al., "Clinical experience with intravenous and oral formulations of the novel histone deacetylase inhibitor suberoylanilide hydroxamic acid in patients with advanced hematologic malignancies," Journal of Clinical Oncology, vol. 24, no. 1, pp. 166-173, 2006.

[35] T. H. Luu, R. J. Morgan, L. Leong et al., "A phase II trial of vorinostat (suberoylanilide hydroxamic acid) in metastatic breast cancer: a California cancer consortium study," Clinical Cancer Research, vol. 14, no. 21, pp. 7138-7142, 2008.

[36] G. R. Blumenschein Jr., M. S. Kies, V. A. Papadimitrakopoulou et al., "Phase II trial of the histone deacetylase inhibitor vorinostat (ZolinzaÜ, suberoylanilide hydroxamic acid, SAHA) in patients with recurrent and/or metastatic head and neck cancer," Investigational New Drugs, vol. 26, no. 1, pp. 81-87, 2008.

[37] Z. An, C. B. Gluck, M. L. Choy, and L. J. Kaufman, "Suberoylanilide hydroxamic acid limits migration and invasion of glioma cells in two and three dimensional culture," Cancer Letters, vol. 292, no. 2, pp. 215-227, 2010.

[38] E. Galanis, K. A. Jaeckle, M. J. Maurer et al., "Phase II trial of Vorinostat in recurrent glioblastoma multiforme: a north central cancer treatment group study," Journal of Clinical Oncology, vol. 27, no. 12, pp. 2052-2058, 2009.

[39] N. Khan and H. Mukhtar, "Cancer and metastasis: prevention and treatment by green tea," Cancer and Metastasis Reviews, vol. 29, no. 3, pp. 435-445, 2010.

[40] J.-D. Liu, S.-H. Chen, C.-L. Lin, S.-H. Tsai, and Y.-C. Liang, "Inhibition of melanoma growth and metastasis by combination with (-)-epigallocatechin-3-gallate and dacarbazine in mice," Journal of Cellular Biochemistry, vol. 83, no. 4, pp. 631-642, 2001.

[41] M. Nihal, C. T. Roelke, and G. S. Wood, "Anti-melanoma effects of vorinostat in combination with polyphenolic antioxidant (-)Epigallocatechin-3-Gallate (EGCG)," Pharmaceutical Research, vol. 27, no. 6, pp. 1103-1114, 2010.

[42] M. Lang, R. Henson, C. Braconi, and T. Patel, "Epigallocatechingallate modulates chemotherapy-induced apoptosis in human cholangiocarcinoma cells," Liver International, vol. 29, no. 5, pp. 670-677, 2009.

[43] H. S. Basu, A. Mahlum, F. Mehraein-Ghomi et al., "Pretreatment with anti-oxidants sensitizes oxidatively stressed human cancer cells to growth inhibitory effect of suberoylanilide hydroxamic acid (SAHA)," Cancer Chemotherapy and Pharmacology, vol. 67, no. 3, pp. 705-715, 2011. 
[44] Y. Li, Y.-Y. Yuan, S. M. Meeran, and T. O. Tollefsbol, "Synergistic epigenetic reactivation of estrogen receptor- $\alpha$ (ER $\alpha)$ by combined green tea polyphenol and histone deacetylase inhibitor in ER $\alpha$-negative breast cancer cells," Molecular Cancer, vol. 9, article 274, 2010.

[45] K.-C. Choi, G. J. Myung, Y.-H. Lee et al., "Epigallocatechin3-gallate, a histone acetyltransferase inhibitor, inhibits EBVinduced B lymphocyte transformation via suppression of RelA acetylation," Cancer Research, vol. 69, no. 2, pp. 583-592, 2009.

[46] S. O. Kim and M. R. Kim, "(-)-Epigallocatechin 3-gallate inhibits invasion by inducing the expression of Raf kinase inhibitor protein in AsPC-1 human pancreatic adenocarcinoma cells through the modulation of histone deacetylase activity," International Journal of Oncology, vol. 42, pp. 349-358, 2013. 


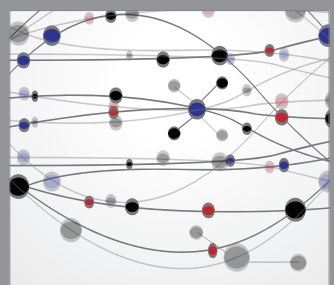

The Scientific World Journal
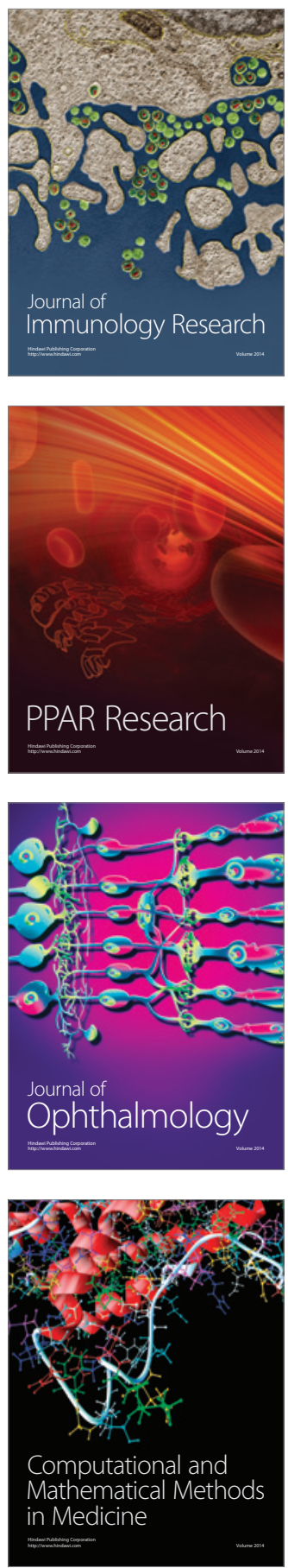

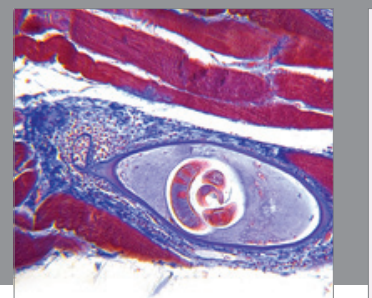

Gastroenterology

Research and Practice
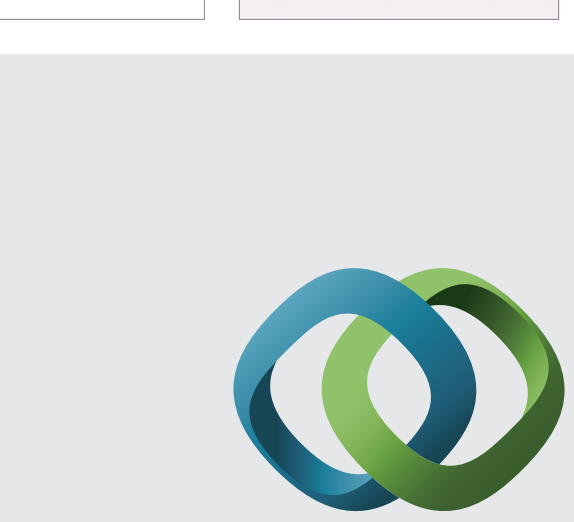

\section{Hindawi}

Submit your manuscripts at

http://www.hindawi.com
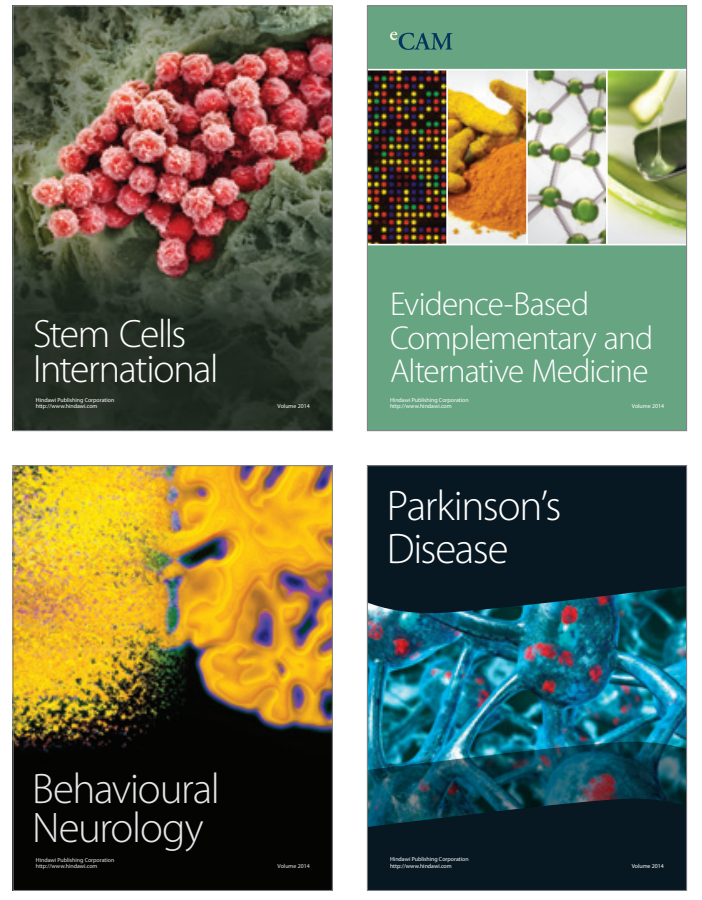
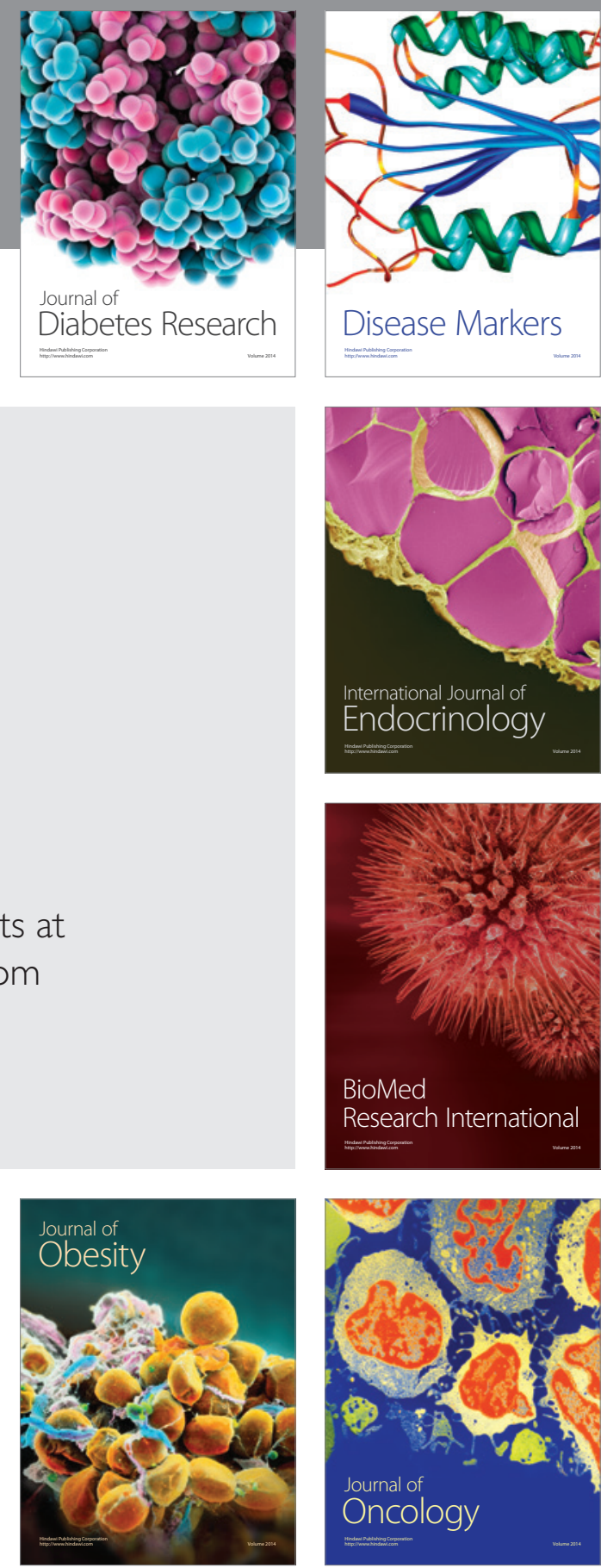

Disease Markers
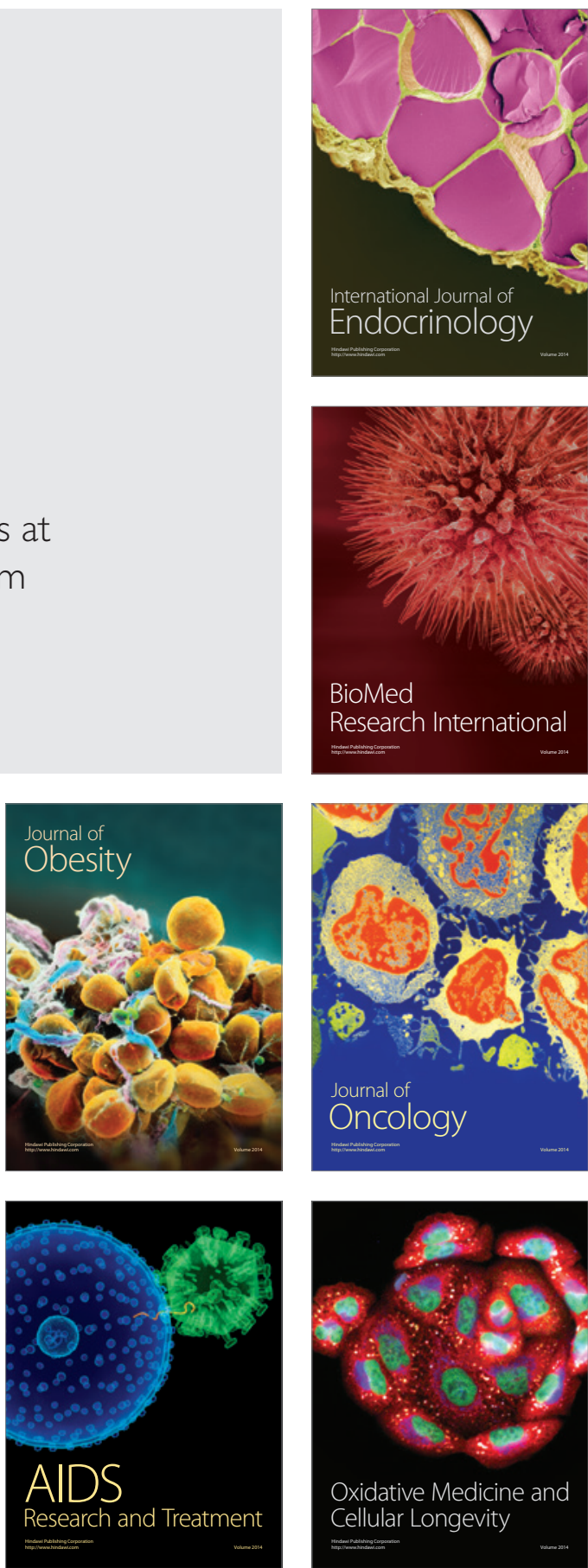